\title{
Hongos patógenos de insectos en Argentina (Zygomycetes: Entomophthorales)
}

\author{
C.C. López Lastra \& A.C. Scorsetti \\ 1 Centro de Estudios Parasitológicos y de Vectores, CEPAVE. Calle 2 N$^{\circ} 584$ (1900) La Plata, Argentina; \\ ccl1@museo.fcnym.unlp.edu.ar
}

Recibido 07-V-2002. Corregido 20-XI-2003. Aceptado 14-X-2004.

\begin{abstract}
Pathogenic fungi of insects from Argentina (Zygomycetes: Entomophthorales). Three species of Entomophthorales entomopathogenic fungi (Zygomycotina: Zygomycetes) have been identified from insects in agricultural crops (Buenos Aires Province, Argentina): Zoophthora radicans Batko (Brefeld); Entomophthora planchoniana Cornu and Pandora gammae (Weiser) Humber. Fungal structure measurements are reported. Rev. Biol. Trop. 54(2): 311-315. Epub 2006 Jun 01.
\end{abstract}

Key words: entomophthorales, fungi, pathogens, insect pests, horticulture, crops, Argentina.

Los estudios sobre hongos patógenos de insectos de interés agrícola citados previamente en la Argentina se refieren a hospederos presentes en cultivos extensivos o pasturas (Fresa 1979, López Lastra 1988 y 1989, Díaz y Lecuona 1995, Lecuona y Díaz 1996, Lecuona 1999) y no existen registros para cultivos hortícolas en invernáculos. El conocimiento de hongos Entomophthorales en la Argentina es muy escaso y fragmentario (Fresa 1971, Lange 1996), algunas citas previas para Sudamérica son las de Aruta et al. (1974) y Aruta et al. (1984) en Chile, y Alzugaray et al. (1999) para Uruguay.

En recolecciones realizadas durante los años 1996, 1998 y 2001 se registró la presencia de tres especies de hongos Entomophthorales patógenos de insectos, que constituyen nuevos registros para la República Argentina y amplían la distribución de este grupo de hongos.

Los insectos fueron recolectados en distintos cultivos y localidades en la provincia de Buenos Aires (Cuadro 1). Para la recolección de los insectos, en el caso de cultivos hortícolas, las plantas fueron revisadas individualmente, mientras que para el cultivo de soja, se utilizó el método de paño (Zeiss y Kubertanz 1993). Los insectos recolectados fueron transportados al laboratorio y se realizó la prospección de las infecciones fúngicas mediante el uso del microscopio estereoscópico. Los insectos infectados presentaron una coloración verde azulado clara o amarillo claro y consistencia arenosa, éstos fueron incubados en cámaras húmedas (cajas de Petri con papel de filtro humedecido con agua destilada estéril) en estufa de cultivo a $20^{\circ} \mathrm{C}$ durante 48 horas. Se realizaron disecciones de los insectos y preparaciones microscópicas de los tejidos infectados que se montaron en azul de algodón lactofenol de Amman y en aceto- orceína 1\%. Los hongos se cultivaron en medio EYSMA (Sabouraud maltosa agar con extracto de levadura + yema de huevo) (Humber 1997). Las especies fúngicas fueron identificadas de acuerdo a Humber (1997) y Balazy (1993).

Las especies que se identificaron se citan a continuación:

Zoophthora radicans Batko (Brefeld) (Fig. 1). 
CUADRO 1

Hongos Entomophthorales en insectos de importancia agricola de la Argentina

\begin{tabular}{|c|c|c|c|c|c|}
\hline $\begin{array}{l}\text { Especie } \\
\text { fúngica }\end{array}$ & Hospedero & $\begin{array}{l}\text { Estado de } \\
\text { desarrollo }\end{array}$ & Planta huésped & $\begin{array}{l}\text { Localidad } \\
\qquad(*)\end{array}$ & Fecha \\
\hline E. planchoniana & Myzus persicae & Adultos & Capsicum annum & Saladillo & 1998 \\
\hline \multirow[t]{2}{*}{ P. gammae } & Rachiplusia nu & Larvas & Glycine $\max$ & Chivilcoy & 1996 \\
\hline & $\begin{array}{l}\text { Anticarsia } \\
\text { gemmatalis }\end{array}$ & & & & \\
\hline \multirow[t]{3}{*}{ Z. radicans } & M. persicae & Ninfas y adultos. & C. annum & Saladillo & 1998 \\
\hline & Tuta absoluta & $\begin{array}{l}\text { Larvas, pupas y } \\
\text { adultos }\end{array}$ & $\begin{array}{l}\text { Lycopersicum } \\
\text { esculentum }\end{array}$ & $\begin{array}{l}\text { Colonia Urquiza, } \\
\text { La Plata }\end{array}$ & 2001 \\
\hline & Epinotia aporema & Larvas & $\begin{array}{l}\text { Lupinus albus } \\
\text { Melilotus albus } \\
\text { Galega officinalis } \\
\text { Vicia faba }\end{array}$ & $\begin{array}{l}\text { Los Hornos, } \\
\text { La Plata }\end{array}$ & 1997,1998 \\
\hline
\end{tabular}

(*) Las localidades pertenecen a la provincia de Buenos Aires.

Hospedero: Tuta absoluta (Meyrick) (Lepidoptera: Gelechidae), larvas, pupas y adultos.

Los conidios fueron medidos en preparaciones "en fresco", y observados con microscopio óptico con contraste de fases. Las medidas expresadas de las estructuras fúngicas observadas son los valores promedio \pm desviación estándar.

Conidios primarios: largo: $16.5( \pm 0.6) \mathrm{x}$ ancho: $6.3( \pm 0.3) \mu \mathrm{m}$. $(\mathrm{n}=20)$.

Conidios secundarios: $9.7( \pm 0.5) \times 4.2( \pm$ 0.32) $\mu \mathrm{m}(\mathrm{n}=20)$.

Capiliconidios: $7.7( \pm 1.3) \times 2.37 \mu \mathrm{m}$ $(n=20)$.

Localidad: Colonia Urquiza, Partido de La Plata, provincia de Buenos Aires, Argentina, en cultivos de tomate (Lycopersicum esculentum), en invernáculos bajo cubierta.

Observaciones: la presencia de Z. radicans fue registrada también en otros insectos hospederos durante Agosto de 1998, en ninfas y adultos de Myzus persicae (Sulzer). Se detectó un máximo porcentaje de infección natural en adultos de $16.5 \%(\mathrm{n}=876)$ y en Epinotia aporema (Wals.) en larvas, con un porcentaje de infección natural de $14.2 \%(\mathrm{n}=7)$ y $18.5 \%(\mathrm{n}=$ 27) en agosto de 1998.
Los aislamientos de $Z$. radicans fueron depositados en la colección micológica del CEPAVE y en la colección de hongos entomopatogénos (ARSEF- USDA- ARS, Ithaca, Nueva York, 14853, USA). Los números de identificación de la colección fueron: ARSEF $n^{\circ} 5814$ y 6917.

Las medidas del material estudiado para este trabajo coinciden con la descripción original (Keller 1991) con respecto a conidios primarios ya que estos son de 15.5-22.5 x 6.3$8.4 \mu \mathrm{m}$, si bien los capiliconidios observados por nosotros son de menor tamaño a los descritos previamente: $17.3-21.8 \times 4.6-5.9 \mu \mathrm{m}$, sin embargo las mediciones fueron realizadas a partir de cultivos y en la presente cita se refiere a material medido en el hospedero. Aruta et al. (1974) citaron Entomophthora sphaerosperma $=Z$. radicans, en larvas de Plutella xylostella y de Copitarsia convicta (Lepidoptera) y en áfidos sobre gramíneas para Chile y las medidas de los conidios primarios presentadas por ellos fueron: $21.2-\mathrm{x}$ 5.9-6.8 $\mu \mathrm{m}$ para lepidópteros y de 14.1- $18 \mathrm{x}$ 13.2-15.8 $\mu \mathrm{m}$ para áfidos.

Entomophthora planchoniana Cornu (Fig. 2). 


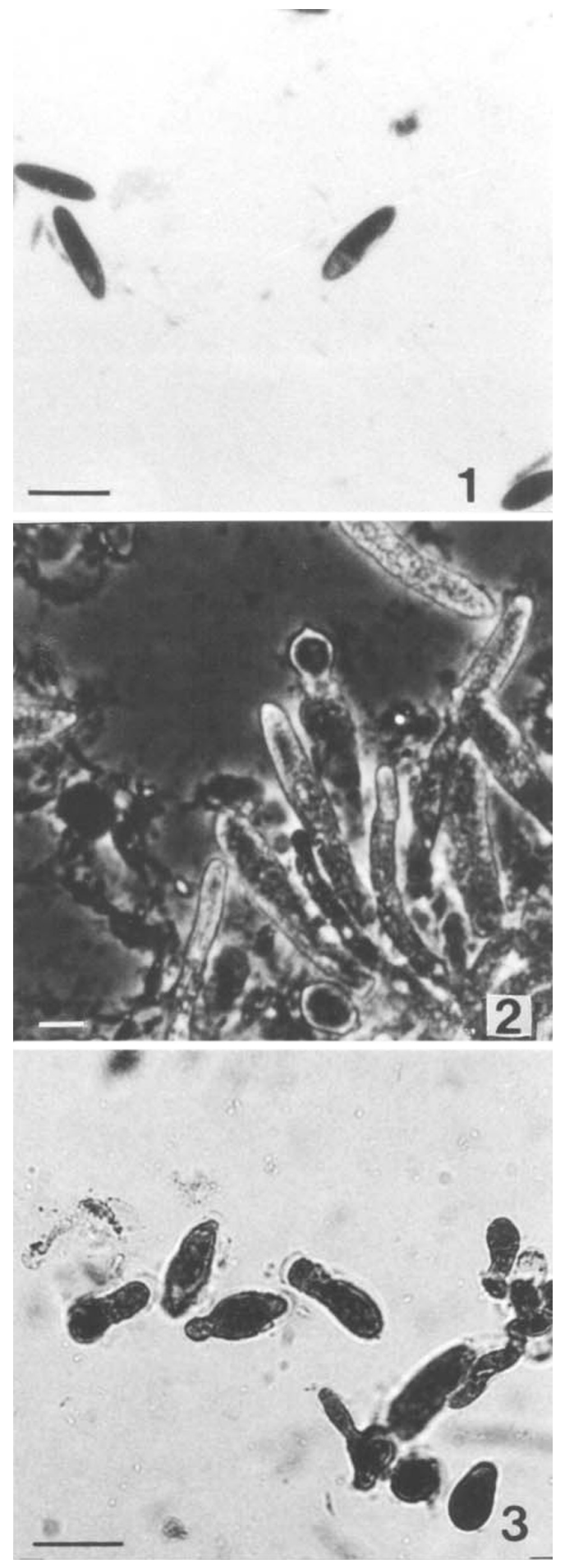

Hospedero: Myzus persicae, (Homoptera: Aphididae) ninfas y adultos.

Los conidios se midieron en preparaciones montadas en azul de algodón - lactofenol de Ammann.

Conidios primarios: $11.4( \pm 0.2) \mu \mathrm{m}$ de diámetro $(\mathrm{n}=20)$.

Localidad: Saladillo, Provincia de Buenos Aires, Argentina, en cultivos de "pimiento" (Capsicum annum) en invernáculos bajo cubierta.

Observaciones: no se detectaron conidios secundarios en el material recolectado. Esta especie no pudo ser aislada en cultivo axénico por lo que ha sido depositada como material fijado en preparados microscópicos en la colección micológica del CEPAVE, número (CEP 100/ ENT98).

En la descripción original de E. planchoniana (Keller 1991), los conidios primarios midieron 17. 5- $18 \times 14-15 \mu \mathrm{m}$, por lo cual el material observado por nosotros presenta conidios levemente menores a los descritos originalmente.

Pandora gammae (Weiser) Humber (Fig. 3).

Hospedero: Anticarsia gemmatalis (Hübner), (Lepidoptera: Noctuidae) larvas de cuarto y quinto estadio.

Localidad: Chivilcoy, Provincia de Buenos Aires, Argentina, en cultivo de soja (Glycine max).

Conidios: $20.3( \pm 0.6) \times 8.2( \pm 0.2) \mu \mathrm{m}$ $(n=20)$.

Fig. 1. Conidios primarios de Zoophthora radicans a partir de larvas de Tuta absoluta. Escala: $10 \mu \mathrm{m}$.

Fig. 2. Conidióforos y conidios primarios de Entomophthora planchoniana, a partir de ninfas de Myzus persicae. c: conidios. Escala: $10 \mu \mathrm{m}$.

Fig. 3. Conidios primarios de Pandora gammae a partir de larvas de Anticarsia gemmatalis. Escala: $10 \mu \mathrm{m}$. 
Observaciones: el material estudiado no pudo ser aislado en cultivo in vitro, por lo que se han depositado preparaciones microscópicas, en la colección micológica del CEPAVE, (CEP 102/ ENT96).

En la Argentina ha sido registrada previamente la presencia de Entomophthorales tales como Erynia gammae (=Pandora gammae) en larvas de Rachiplusia nu sobre cultivo de girasol en Necochea, provincia de Buenos Aires (Fresa 1979) sin presentar medidas de estructuras y Entomophaga grylli en adultos de Dichroplus sp en las localidades de General Villegas (Fresa 1971) y de Villa Sauri (partido de Adolfo Alsina) (Lange 1996).

Las medidas de los conidios primarios del material estudiado coinciden con la descripción original, $(19-21 \times 7.5-10 .-5 \mu \mathrm{m})$. Las cigosporas son de mucho mayor tamaño, 4547 x $41-44.4 \mu \mathrm{m}$ que el material estudiado en este trabajo, por lo cual podríamos considerar que en nuestro material podrían ser probablemente cigosporas inmaduras o en formación o bien cuerpos hifales en proceso de formación de cigosporas.

El presente trabajo contribuye a ampliar la distribución geográfica de especies fúngicas entomopatógenas, de su espectro hospedero y de nuevos cultivos en los cuales se han registrado hongos Entomophthorales.

\section{AGRADECIMIENTOS}

A Richard A. Humber por su valiosa colaboración en la confirmación e identificación de las especies fúngicas. A Gloria Rojas y Pablo Zarragoicoechea por su colaboración con la recolección del material. A Victoria Micieli y Gustavo Rossi por la lectura crítica del manuscrito. C.C. López Lastra es Investigador Científico del CONICET (Consejo Nacional de Investigaciones Científicas y Técnicas, Argentina).

\section{RESUMEN}

Se informa de tres especies de hongos Entomophthorales en insectos encontrados en cultivos agrícolas en la Provincia de Buenos Aires, Argentina: Zoophthora radicans Batko (Brefeld); Entomophthora planchoniana Cornu y Pandora gammae (Weiser) Humber. Se incluyen las medidas de las estructuras fúngicas.

Palabras clave: entomophthorales, hongos, patógenos, insectos plaga, horticultura, cultivos, Argentina.

\section{REFERENCIAS}

Alzugaray, R., M.S. Zerbino, S. Stewart, A. Ribeiro \& J. Eilenberg.1999. Epizootiología de hongos Entomophthorales. Uso de Zoophthora radicans (Brefeld) Batko (Zygomycotina: Entomophthorales) para el control de Epinotia aporema (Wals.) (Lepidoptera: Tortricidae) en Uruguay. Rev. Soc. Ent. Arg. 58(1-2): 307-311.

Aruta, C.M., R.Ll. Carrillo \& S.M. González. 1974. Determinación para Chile de Hongos entomopatógenos del género Entomophthora. I. Agro Sur 2(2): 62-70.

Aruta, C.M., R.Ll. Carrillo \& J.A. Montealegre. 1984. Determinación para Chile de hongos del Orden Entomophthorales (Zygomycetes). Agro Sur 12(1): $36-42$.

Balazy, S. 1993. Flora of Poland Fungi (Mycota) Entomophthorales. Polish Acad. of Sciences W. Szafer. Inst. Botany Kraków vol XXIV. p. 1-356.

Díaz, B.M. \& R.E. Lecuona. 1995. Evaluación de cepas nativas del hongo entomopatógeno Beauveria bassiana Bals. (Vuill.) (Deuteromycotina) como base para la selección de bioinsecticidas contra el barrenador Diatraea saccharalis (F.). Agriscientia 12: 33-38.

Fresa, R. 1971. El hongo Entomophthora grylli en tucuras. Revista de Investigaciones Agropecuarias, INTA Serie Patología Vegetal. 7(2): 83-88.

Fresa, R. 1979. Hongos entomopatógenos observados en larvas de lepidópteros perjudiciales para cultivos de la República Argentina. I.D.I.A. 373: 149-155.

Humber, R. 1997. Fungi identification. pp 153-185. In L. Lacey. (ed.). Manual of Techniques in Insect 
Pathology. Academic, San Diego, California, EEUU.

Keller, S. 1991. Arthropod- pathogenic Entomophthorales of Switzerland II. Erynia, Eryniopsis, Neozygites, Zoophthora and Tarichium. Sydowia 43: 39-122.

Lange, C.E. 1996. Melanoplinos (Orthoptera: Acrididae) afectados por micosis en la provincia de Buenos Aires, Argentina. Rev. Soc. Ent. Arg. 55(1-4): 107-109.

Lecuona , R.E. \& B.M. Díaz. 1996. Compatibilidad de dos cepas del hongo entomopatógeno Beauveria bassiana con distintos insecticidas químicos. R.I.A 26(1): 77-82.

Lecuona, R.E. 1999. Control microbiano con hongos entomopatógenos. Rev. Soc. Ent. Arg. 58(1-2): 301-306.
López Lastra, C.C. 1988. Nuevas especies de insectos hospederos para el hongo Beauveria bassiana (Bals.) Vuill. (Deuteromycotina: Hyphomycetes) en Argentina y consideraciones sobre su patogenia. Rev. Fac. Agr. UNLP Tomo LXIV (entregas 1 y 2): 42-46.

López Lastra, C.C. 1989. Nuevos hospedadores para los hongos entomopatógenos Metarhizium anisopliae (Metchnikoff) Sorokin y Verticillium lecanii (Zimmerman) Viegas, en la República Argentina. Rev. Soc. Ent. Arg. 47(1): 94.

Zeiss, M.R. \& T.H. Klubertanz. 1993. Sampling program for Soybean Arthropods. p. 539-602. In Pedigo \& Buntin (eds.). Handbook of Sampling Methods for Arthropods in Agriculture, CRC, Boca Raton, Florida, EEUU. 
